\title{
Breast Cancer News from ASCO 2013
}

\author{
Chair: Peter Dalla \\ Participants: Cornelia Liedtke ${ }^{\mathrm{b}}{\text { Brigitte } \text { Rack }^{\mathrm{c}} \text { Florian Schütz }}^{\mathrm{d}}$ Elmar Stickeler \\ aFrauenklinik, Gynäkologisches Krebszentrum/Brustzentrum, Städtisches Klinikum Lüneburg,

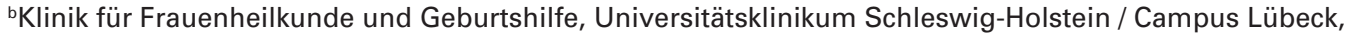 \\ cKlinik und Poliklinik für Frauenheilkunde und Geburtshilfe, Klinikum Innenstadt, Ludwig-Maximilians-Universität München, \\ dUniversitätsfrauenklinik Heidelberg, \\ eUniversitätsfrauenklinik, Brustzentrum, Universitätsklinikum Freiburg i.Br., Germany
}

\section{Question 1: What Was Your Personal Highlight of ASCO 2013 in Breast Cancer?}

Liedtke: Compared to previous meetings this year's ASCO Annual Meeting did present with a number of interesting data rather than one particular highlight. These aspects comprise data regarding adjuvant therapy in HER2-positive breast cancer, axillary staging, and optimization of adjuvant chemoand endocrine therapy. Since axillary staging in general has recently been an issue of debate in both the adjuvant and the neoadjuvant setting, I was particularly interested to see the data regarding the AMAROS trial presented by Rutgers et al., demonstrating equal efficacy for axillary dissection and axillary radiation in patients with sentinel lymph node-positive breast cancer.

Rack: The aTTom trial confirming prolonged benefit from extended adjuvant tamoxifen treatment for 10 years after primary diagnosis was my personal highlight. 10 years of tamoxifen resulted in an improvement of $15 \%$ for breast cancer mortality compared to only 5 years of treatment.

Schütz: Two studies have shown us that our knowledge about classical medication in early breast cancer can still be optimized: in the GeparSixto trial presented by Gunter von Minckwitz the addition of carboplatin to an anthracycline/ taxane sequence improved pathological complete response (pCR) in triple negative breast cancer (TNBC) up to $60 \%$. However, only $52 \%$ of the patients were able to take the planned 6 cycles, mostly due to toxicity. Furthermore, in endocrine therapy the 10-year follow up of the aTTom trial was presented. With regard to the recently published ATLAS trial (Lancet, 2013) it was shown in more than 18,000 patients that 10 years of tamoxifen reduces recurrence rate as well as mortality over the following years. However, patients have to deal with a higher incidence of endometrial cancer.
Stickeler: The principle of a dual blockade of the HER2 receptor pathway was my highlight. Even if most of these innovative findings were already reported at the San Antonio Breast Cancer Symposium the evidence of the superiority is now striking and the procedure is becoming clinical practice.

\section{Question 2: uPA/PAl1 vs. Oncotype DX vs. Endopredict etc. - What Is Your Preferred Concept to Differentiate in Intermediate-Risk Patients?}

Liedtke: Since none of these tests has yet demonstrated superiority this question is difficult to answer. In our department, we are currently using particularly uPA/PAI-1 or Endopredict. However, I am convinced that recommending chemotherapy to a patient with intermediate-risk breast cancer should depend on both disease prognosis and prediction of response (to endocrine therapy). Therefore, I strongly support the ongoing ADAPT trial which stratifies patients to either receive or not receive chemotherapy depending on their response to short-term endocrine therapy (measured by sequential measurement of KI-67). Patients should be strongly encouraged to participate in this trial if possible.

Rack: Oncotype DX has shown prognostic and predictive relevance in several retrospective analyses by different research groups. An advantage is also that the test can be performed on formalin-embedded tissue and is therefore available in most clinical settings.

Schütz: Unfortunately there is no prospective trial available that has proven the predictive nature of any test for modern systemic treatment. We have decided to use the proliferation marker Ki67 to decide whether chemotherapy is necessary or not. Although there is still a lack of information we have used Oncotype DX a few times over the last years.

\section{KARGER}

Fax +497614520714

Information@Karger.com

www.karger.com (c) 2013 S. Karger GmbH, Freiburg

1661-3791/13/0084-0307\$38.00/0

Accessible online at:

www.karger.com/brc
Prof. Dr. med. Peter Dall

Frauenklinik

Gynäkologisches Krebszentrum/Brustzentrum

Städtisches Klinikum Lüneburg gGmbH

Bögelstraße 1, 21339 Lüneburg, Germany

peter.dall@klinikum-lueneburg.de 
Stickeler: Since uPA/PA1 was evaluated in a truly prospective setting I prefer this test momentarily. However, the evidence for Oncotype DX is strong since the data were generated in prospectively designed trials in a so-called retrospective-perspective manner.

\section{Question 3: Is There a Standard in Adjuvant Treatment of Triple Negative Breast Cancer? Are There Several?}

Liedtke: Since chemotherapy is indicated in the majority of patients with TNBC at the time of diagnosis, a neoadjuvant approach is usually recommended. At this year's ASCO, the German GeparSixto trial conducted by the German Breast Group (GBG) has demonstrated significant efficacy of a taxane-anthracycline chemotherapy regimen in combination with weekly carboplatin particularly among patients with TNBC. Although a pCR rate of about $60 \%$ could be observed among patients with TNBC, the authors observed a significant range of toxicities with $44 \%$ of patients experiencing at least 1 serious adverse event. Therefore, use of this regimen in clinical routine cannot yet be recommended.

Rack: According to present guidelines, adjuvant chemotherapy should be performed irrespective of the molecular tumor subtype. However, the Gunter von Minckwitz presented promising data from the GeparSixto trial in the TNBC subgroup, showing an increase of $\mathrm{pCR}$ with a platinum based treatment. This could be a new option for TNBC. However, the increased toxicity of this regimen with only $60 \%$ of patients being able to complete chemotherapy has to be kept in mind.

Schütz: I don't think that we can define one standard treatment for TNBC. We try to personalize the systemic treatment by discussing every patient in our pretherapeutic tumor conference. We try to treat all TNBC patients in the neoadjuvant setting to observe tumor regression. Therefore we mainly use the classical TAC regimen. However, if we find nodal involvement after primary chemotherapy we discuss a post-neoadjuvant systemic treatment, e.g. with carboplatin based chemotherapy, in this high-risk group.

Stickeler: So far there is no standard for TNBC. However, the treatment guidelines of the AGO suggest to stay within the published adjuvant treatment protocols. The data from the GeparSixto trial presented in Chicago support the hypothesis that patients diagnosed with this clinical subtype will profit from the addition of carboplatin in the neoadjuvant setting. These phase II data are promising but need further confirmation.

\section{Question 4: What Does Modern Radiotherapy Look Like Now and in the Next Years?}

Liedtke: The efficacy of radiotherapy in enhancing locoregional control among patients with early breast cancer is well established. The results of the AMAROS trial suggest that in certain clinical scenarios (axillary) radiation provides an option to replace axillary dissection with equal efficacy but less toxicity. However, retrospective analyses suggest that both, risk of locoregional recurrence and efficacy of adjuvant radiotherapy strongly depend on molecular subtypes. I believe that locoregional control through radiotherapy may eventually be decided on in an equally personalized fashion as we currently decide on adjuvant systemic therapy.

Rack: As efficacy and side effects are comparable, hypofractionated radiotherapy regimens will probably be used more frequently in the future. Irradiation of the axilla might be an option in selected cases (AMAROS trial). Intraoperative boost or intraoperative radiotherapy as only radiation treatment for low-risk patients is becoming a convenient alternative for many patients.

Schütz: Over the next years we will observe a trend towards taking back the extent of radiotherapy as it has happened in the surgical treatment as well. Intraoperative radiotherapy (IORT) as well as partial breast irradiation in defined subgroups may be as effective as whole breast irradiation.

Stickeler: In my opinion hypofractionation will be the first step towards an innovative safe, less toxic, and faster irradiation concept.

\section{Question 5: What Is the Greatest Innovation in the Treatment of Metastatic Breast Cancer?}

Liedtke: To date the most interesting proof of concept in the treatment of metastatic breast cancer is to reverse resistance against endocrine therapy and/or HER2-targeted agents through the use of molecularly targeted agents such as the mTOR inhibitor everolimus in the BOLERO-2 and BOLERO-3 clinical trials, respectively. These data show that molecular manipulation of cancer cells is possible and effective. However, this approach also poses several questions, such as at which disease stage (i.e. curative vs. metastatic) these agents should be used or whether to continue these substances upon diagnosis of disease progression. These questions will need to be answered soon and accordingly trials are currently ongoing. 
Rack: There was not much new in metastatic breast cancer. The BOLERO-3 trial was presented comparing daily everolimus plus weekly trastuzumab and vinorelbine in trastuzumabresistant, advanced breast cancer. O'Regan could show a statistically significant improvement of progession free survival from 5.78 to 7 months. However, the clinical benefit in view of HER2-targeted agents, i.e. dual blockade, and TDM-1 is still unclear.

Schütz: We have made significant improvements in the systemic therapy of metastatic breast cancer over the last years. However, over time most of the patients will experience a resistance against new therapies. Drugs which can modify that resistance like will become more and more important in this setting. The mTOR-inhibitor everolimus is an example for these new drugs as it may re-sensitize tumor cells for an endocrine therapy. Furthermore the combination of targeted therapies and 'old school anticancer treatment', such as TDM-1, is of special interest. This combination of an antibody (against HER2) and a cytotoxic molecule (emtansine) brings the toxic agent only inside those cells that expresses HER2 - a fascinating new way to treat HER2-positive breast cancer.

Stickeler: The development of highly effective concepts to overcome resistance to HER2-targeted drugs with the innovative dual blockade as well as the specific delivery of cytotoxics by an antibody guided approach as well as the targeting of endocrine resistance by new pathway inhibitors (e.g. mTOR inhibition) are great innovations.

\section{Participants}

PD Dr. med. Cornelia Liedtke

Klinik für Frauenheilkunde und Geburtshilfe

Universitätsklinikum Schleswig-Holstein / Campus Lübeck

Ratzeburger Allee 160

23538 Lübeck

cornelia.liedtke@uksh.de

PD Dr. med. Brigitte Rack

Klinik und Poliklinik für Frauenheilkunde und Geburtshilfe,

Klinikum Innenstadt

Ludwig-Maximilians-Universität

Maistrasse 11

80337 München

Brigitte.Rack@med.uni-muenchen.de

Prof. Dr. med. Florian Schütz

Universitätsfrauenklinik Heidelberg

Voßstr. 9, 69115 Heidelberg

Florian.Schuetz@med.uni-heidelberg.de

Prof. Dr. med. Elmar Stickeler

Universitätsfrauenklinik

Brustzentrum Freiburg

Universitätsklinikum Freiburg

Hugstetter-Str. 55, 79106 Freiburg i.Br.

elmar.stickeler@uniklinik-freiburg.de 